\title{
Successful transition to the QFT-PLUS assay, but more is needed for diagnosis of latent tuberculosis infection
}

\author{
Eun Hye Lee ${ }^{1}$ and Young Ae Kang ${ }^{2,3}$
}

${ }^{1}$ Division of Pulmonology, Allergy and Critical Care Medicine, Department of Internal Medicine, Yongin Severance Hospital, Yonsei University College of Medicine, Yongin; ${ }^{2}$ Division of Pulmonology, Department of Internal Medicine, Severance Hospital, Yonsei University College of Medicine, Seoul; ${ }^{3}$ Institute of Immunology and Immunological Diseases, Yonsei University College of Medicine, Seoul, Korea
Received: February 7, 2020 Accepted: February 15, 2020

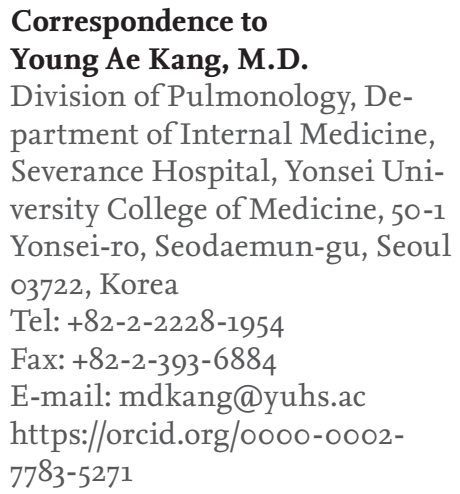

\section{See Article on Page 383-391}

South Korea has an intermediate burden of tuberculosis (TB). The annual incidence of TB was reported to be 66 per 100,000 in 2018, and TB remains a serious public health problem [1]. Latent tuberculosis infection (LTBI) is the state in which humans are infected with Mycobacterium tuberculosis without any clinical symptoms, radiological abnormality, or microbiological evidence. For effective TB control, South Korean guidelines recommended treatment of LTBI patients at high risk of progressing to active TB, including immunocompromised patients and those with close contact to patients with active TB [2]. Tuberculin skin tests and/or interferon- $\gamma($ IFN- $\gamma$ ) release assays (IGRAs) are used to diagnose LTBI.

The QuantiFERON-TB Gold In-Tube (QFT-GIT) test is a widely used IGRA test. A new version, QuantiFERON-TB Gold PLUS (QFT-PLUS), was introduced in 2015. QFT-PLUS contains two TB-specific antigen tubes. The TB1 tube contains long peptides derived from early secretory antigenic target- 6 (ESAT-6) and culture filtrate protein 10 (CFP10) (TB-7.7, which was present in the previous QFT-GIT version, was removed), and is designed to induce a specific $\mathrm{CD}_{4}$ T-cell response. The TB2 tube contains the same long peptides as the TB1 tube as well as short peptides that stimulate IFN- $\gamma$ production by $\mathrm{CD}_{4}$ and $\mathrm{CD} 8 \mathrm{~T}$ cells [3]. In a recent meta-analysis, the pooled sensitivity and specificity values of QFT-PLUS for active TB were 0.94 and 0.96 , respectively. For LTBI diagnosis, the sensitivity and specificity values were 0.91 and 0.95 , respectively [4].

In the latest issue of the Korean Journal of Internal Medicine, Kim et al. [5] reported a comparative evaluation of QFT-PLUS and QFT-GIT for the diagnosis of TB infection in South Korea. In their study, QFT-PLUS and QFT-GIT had highly comparable results for TB infection diagnosis: $91.2 \%$ agreement and a Cohen's $\kappa$ of 0.807 . In another recent study in South Korea that compared QFT-PLUS and QFT-GIT for the diagnosis of LTBI among 317 immunocompromised patients [6], 92 (29.0\%) and $88(27.8 \%)$ patients were diagnosed with LTBI by QFT-GIT and QFT-PLUS, respectively. The rate of concordance between QFT-GIT and QFT-PLUS was 93.7\% ( $\kappa$ value, 0.860 ). It has been suggested that a greater response to TB2 than TB1 would be associated with an increased mycobacterial load and recent TB infection [7]. However, according to Kim et al. [5], the median value 
of TB2 minus TB1 in QFT-PLUS-positive subjects did not differ significantly between the high probability of recent infection group (active TB and TB contact, $\mathrm{n}=19$ ) and the low probability of recent infection group (biologic therapy and other diseases, $\mathrm{n}=25$ ). This unexpected result might have been due to the small number of patients in the high-risk TB infection group and/or the presence of recent LTBI-infected subjects in the low-risk TB infection group owing to casual contact with active TB patients in South Korea. Kim et al. [5] concluded that two QFT-TB IGRAs have comparable performance, but the results could not confirm the diagnostic advantage of QFT-PLUS over QFT-GIT.

The current tests for LTBI, tuberculin skin tests and IGRAs, provide evidence of an immune-memory response to M. tuberculosis. We diagnose LTBI based on QFT-GIT, for which the positive predictive values for incident TB over a 2 -year period are reportedly $1 \%$ to $6 \%[8,9]$. The poor predictive value of available diagnostics for LTBI hampers the implementation of preventive therapy for LTBI as part of a TB control strategy. To overcome this obstacle, new diagnostics that can differentiate incipient TB from persistent infection are needed. The poor predictive value for incident TB even in the high-risk group [9] shows that IGRAs cannot differentiate between persistent infection and incipient TB. Although we do not have long-term data on the predictive power of the QTF-PLUS for active $\mathrm{TB}$, this test is unlikely to achieve the target for predicting incipient TB. The need for better predictive biomarkers for incident TB has prompted the WHO to define a target product profile for incipient TB diagnostics, mandating a minimum sensitivity and specificity of $75 \%$ and an optimal sensitivity and specificity of $90 \%$ over a 2 -year period. These minimum criteria are based on achieving a positive predictive value of $5.8 \%$, when assuming $2 \%$ pre-test probability, to improve on the predictive power of existing tests [10].

In summary, although it was difficult to differentiate between the $\mathrm{TB}_{1}$ and $\mathrm{TB}_{2}$ antigen response based on whether the patients have a recent TB infection or immunocompromised status due to the small number of patients, Kim et al. [5] suggested that the performance of QFT-PLUS is comparable to that of QFT-GIT in South Korea. Other predictive biomarkers and/or diagnostic methods are needed to identify LTBI patients at high risk of developing active TB.

\section{Conflict of interest}

No potential conflict of interest relevant to this article was reported.

\section{REFERENCES}

1. World Health Organization. Global Tuberculosis Report 2019. Geneva (CH): World Health Organization, 2019.

2. Joint Committee for the Revision of Korean Guidelines for Tuberculosis, Korea Centers for Disease Control and Prevention. Korean Guidelines for Tuberculosis. 3rd ed. Seoul (KR): Joint Committee for the Revision of Korean Guidelines for Tuberculosis, 2017.

3. Nikolova M, Markova R, Drenska R, et al. Antigen-specific CD4- and CD8-positive signatures in different phases of Mycobacterium tuberculosis infection. Diagn Microbiol Infect Dis 2013;75:277-281.

4. Sotgiu G, Saderi L, Petruccioli E, et al. QuantiFERON TB Gold Plus for the diagnosis of tuberculosis: a systematic review and meta-analysis. J Infect 2019;79:444-453.

5. Kim SH, Jo KW, Shim TS. QuantiFERON-TB Gold PLUS versus QuantiFERON- TB Gold In-Tube test for diagnosing tuberculosis infection. Korean J Intern Med 2019;35:383-391.

6. Ryu MR, Park MS, Cho EH, et al. Comparative evaluation of QuantiFERON-TB Gold In-Tube and QuantiFERON-TB Gold Plus in diagnosis of latent tuberculosis infection in immunocompromised patients. J Clin Microbiol 2018;56:e00438-18.

7. Pieterman ED, Liqui Lung FG, Verbon A, et al. A multicentre verification study of the QuantiFERON(R)-TB Gold Plus assay. Tuberculosis (Edinb) 2018;108:136-142.

8. Abubakar I, Drobniewski F, Southern J, et al. Prognostic value of interferon- $\gamma$ release assays and tuberculin skin test in predicting the development of active tuberculosis (UK PREDICT TB): a prospective cohort study. Lancet Infect Dis 2018;18:1077-1087.

9. Diel R, Loddenkemper R, Nienhaus A. Predictive value of interferon- $\gamma$ release assays and tuberculin skin testing for progression from latent TB infection to disease state: a meta-analysis. Chest 2012;142:63-75.

10. Gupta RK, Turner CT, Venturini C, et al. Concise whole blood transcriptional signatures for incipient tuberculosis: a systematic review and patient-level pooled meta-analysis. Lancet Respir Med 2020 Jan 17 [Epub]. https:// doi.org/10.1016/S2213-2600(19)30282-6. 